\title{
La structure du temps est-elle indécidable? Sous-détermination et structure du temps chez Newton-Smith
}

\author{
Vincent Ardourel \\ Université Paris IV — Paris-Sorbonne
}

Version avant correction des épreuves.

ARDOUREL, Vincent (2014). La structure du temps est-elle indécidable ? Sousdétermination et structure du temps chez Newton-Smith. Dialogue, Cambridge University Press, vol. 53 (4), p. 623-649. DOI: 10.1017/S0012217314001097

\section{RÉSUMÉ :}

Dans La structure du temps, Newton-Smith avance la thèse selon laquelle il est impossible de décider si la structure du temps est continue ou simplement dense. Le but de cet article est d'examiner dans quelle mesure la thèse de Newton-Smith est justifiée. D'un côté, je soutiens que l'argumentation qu'il propose est insuffisamment convaincante. D'un autre côté, je montre comment pallier cette critique et, dans ce nouveau contexte, je propose une révision des conséquences qu'il tire de son résultat d'indécidabilité.

\section{ABSTRACT :}

In his book The Structure of Time, Newton-Smith claims that we cannot decide whether the structure of time is continuous or merely dense. I discuss here to what extent he is right. On the one hand, I show that Newton-Smith fails to provide strong enough arguments to defend his claim. On the other hand, I suggest first how to overcome this issue. Then, I argue that we have to reconsider the consequences that Newton-Smith draws from his undecidability result. 


\section{Introduction}

Aux chapitres VI et X de La structure du temps, Newton-Smith avance la thèse selon laquelle il est impossible de déterminer la structure du temps. Plus précisément, il est impossible de décider si la structure du temps est continue ou simplement dense. Par structure continue du temps, il entend la conception du temps comme un ensemble d'instants isomorphe à l'ensemble des nombres réels et par structure dense, celle d'un ensemble d'instants isomorphe à l'ensemble des nombres rationnels. Selon lui, les deux énoncés le temps est continu et le temps est simplement dense sont sous-déterminés par les données empiriques. Il est impossible de trancher à l'aide d'une expérience entre deux théories physiques dont l'une supposerait la continuité du temps et l'autre sa simple densité. Il s'agit d'un «résultat d'indécidabilité» (Newton-Smith, 1980, p. 126) qu'il est possible d'interpréter de deux manières. D'un côté, il indique une limite à la connaissance de la structure temps. La structure du temps est soit continue, soit simplement dense mais elle reste impossible à déterminer. D'un autre côté, en faveur duquel Newton-Smith semble s'engager, les énoncés sur la structure du temps ne doivent pas être interprétés comme des énoncés empiriques mais comme des cadres théoriques au sein desquels les théories scientifiques sont construites.

Le résultat d'indécidabilité avancé par Newton-Smith n'a pas fait l'objet d'une attention soutenue de la part de la communauté philosophique ${ }^{1}$. Le but de cet article est d'examiner dans quelle mesure la thèse Newton-Smith est justifiée. D'un côté, je soutiens que l'argumentation proposée par Newton-Smith n'est pas suffisamment convaincante. Il avance la possibilité de construire des théories physiques empiriquement équivalentes reposant sur des représentations différentes du temps. Mais la construction qu'il propose conduit à des théories physiques inutilisables et, à ce titre, ne permet pas de soutenir efficacement sa thèse. D'un autre côté, je montre comment surmonter cette critique et construire une mécanique classique qui repose sur une représentation dense du temps. Dans ce nouveau contexte, je discute l'interprétation du résultat d'indécidabilité proposée par Newton-Smith et plaide en faveur d'une neutralité de la représentation du temps relativement à la structure supposée du temps. C'est la représentation elle-même du temps comme continu, et non sa structure continue, qui doit être interprétée comme un cadre théorique pour décrire les phénomènes.

La section 1 est consacrée à l'examen de la construction proposée par Newton-Smith d'une mécanique classique reposant sur une représentation dense du temps et empiriquement équivalente à la mécanique traditionnelle. Dans la section 2, je montre pourquoi cette construction n'est pas satisfaisante et propose de la modifier en conséquence. Dans la section 3, j'examine dans ce nouveau contexte l'interprétation que propose Newton-Smith de

\footnotetext{
${ }^{1}$ C'est dans le contexte de la sous-détermination des théories par l'expérience que l'on trouve des discussions du résultat d'indécidabilité de Newton-Smith : (Quine, 1979), (Hesse, 1980, p. 8-9), (Bergström, 1984, p. 351-352) et (Norton, 2008, p. 26). Mais ces discussions sont elliptiques et aucune d'entre elles ne propose un examen des arguments de Newton-Smith.
} 
l'équivalence empirique entre la mécanique classique continue et la mécanique dense et, dans la section 4 , je propose une révision de son interprétation.

\section{La représentation du temps comme dense}

C'est dans le contexte du débat sur la sous-détermination des théories que Newton-Smith envisage la possibilité de représenter le temps comme simplement dense (Newton-Smith, 1978 ; Newton-Smith, 2000). La sous-détermination forte des théories (SFT) est la thèse selon laquelle, pour toute théorie, il existe une théorie rivale incompatible et empiriquement équivalente avec toutes les données actuelles et possibles. Il s'agit d'une thèse majeure contre le réalisme scientifique puisque aucune théorie ne peut, dans ce cas, être tenue pour vraie sur la base des données empiriques, même approximativement, puisqu'il existe au moins en principe une théorie rivale incompatible. Au vu des débats en philosophie des sciences, selon Newton-Smith, « la seule conclusion que nous pouvons tirer est que la SFT est, au mieux, une conjecture infondée, hautement spéculative » (Newton-Smith, 2000, p. 535). Cependant, en dépit de cette conclusion négative, il suggère une voie possible pour réhabiliter la thèse de la sous-détermination : " même s'il n'y a pas d'argument convaincant en faveur de la SFT, on a proposé d'étonnantes conjectures selon lesquelles certains aspects de l'espace et du temps sont susceptibles de sous-détermination forte. » (Newton-Smith, 2000, p. 535). Newton-Smith fait ici référence au cas qu'il a lui-même envisagé en 1978 dans «La sous-détermination des théories par les données» puis, en 1980, dans La structure du temps, et qui fait l'objet de cet article. Il s'agit du cas selon lequel la continuité du temps et sa simple densité ne peuvent pas être distinguées empiriquement.

Supposons que deux théories physiques, l'une représentant le temps comme continu et l'autre comme simplement dense, soient empiriquement équivalentes. Autrement dit, il ne serait pas possible de distinguer les prédictions issues de ces deux théories à l'aide de mesures physiques. Dans ces conditions, selon Newton-Smith, nous ne pourrions pas décider si la structure du temps est continue ou simplement dense :

La science représente traditionnellement l'espace et le temps comme des grandeurs non seulement denses mais aussi continues. Cela signifie que les points d'un intervalle de temps ou d'espace sont représentés avec de nombres réels et non avec des nombre rationnels. Cependant, comme tous les résultats de mesure ne possèdent qu'un nombre fini de décimales, les résultats d'observations sont toujours des nombres rationnels. Cela conduit à la conjecture selon laquelle nous avons le choix de considérer l'espace et le temps comme continus ou simplement denses. (Newton-Smith, 2000, p. 535)

Newton-Smith s'appuie sur l'exemple de la mécanique classique, une théorie physique qui utilise traditionnellement une représentation continue du temps. Il avance la possibilité de 
construire une autre mécanique classique, semblable à la mécanique de Newton, mais reposant sur une représentation dense du temps. Il nomme cette nouvelle théorie la mécanique de Notwen et soutient qu'elle conduit à des prédictions théoriques qui ne peuvent être distinguées empiriquement de celles de la mécanique de Newton.

Dans cette section, je commence par clarifier la distinction entre les notions de densité et de continuité (section 1.1). J'examine ensuite la construction de la mécanique dense proposée par Newton-Smith (section 1.2) avant d'expliciter en quel sens elle est empiriquement équivalente à la mécanique continue de Newton (section 1.3).

\subsection{Densité et continuité}

La possibilité de remplacer la représentation continue du temps en mécanique classique par une représentation du temps comme dense est envisagée par Newton-Smith de la manière suivante. Il s'agit de remplacer la représentation du temps à l'aide du paramètre $t$ défini sur l'ensemble des nombres réels par un paramètre $t$ défini sur l'ensemble des nombres rationnels ${ }^{2}$. En effet, l'ensemble des nombres rationnels est un ensemble dense, c'est-à-dire qu'entre deux nombres rationnels aussi proches que l'on veut, il existe toujours un nombre rationnel. Ainsi, en représentant le temps par un paramètre rationnel, quels que soient les instants $t_{1}$ et $t_{2}$ tels que $t_{1}<t_{2}$, il existe toujours un instant $t_{3}$ tel que $t_{1}<t_{3}<t_{2}$.

La représentation dense du temps se distingue à la fois d'une représentation discrète et d'une représentation continue. D'un coté, selon Newton-Smith, une représentation discrète du temps consiste à utiliser un paramètre $t$ défini sur l'ensemble des nombres entiers. Dans ce cas, entre deux instants quelconques il n'existe pas forcément d'instants compris entre les deux $^{3}$. La représentation dense est à ce titre plus riche que la représentation discrète. D'un autre côté, la continuité est une notion plus forte que la densité. L'ensemble des nombres réels est non seulement dense mais aussi complet, c'est-à-dire que la limite de toute suite de nombres réels est un nombre réel. Au contraire, la limite d'une suite de nombre rationnels n'est pas forcément un nombre rationnel ${ }^{4}$. La différence entre la continuité de l'ensemble des nombres réels et la simple densité des nombres rationnels peut aussi être caractérisée en termes de cardinalité. Alors que l'ensemble des nombres rationnels est dénombrable, c'est-à-

\footnotetext{
${ }^{2}$ L'ensemble des nombres rationnels, noté $\boldsymbol{Q}$, est l'ensemble des nombres qui peuvent s'écrire comme un rapport de deux nombres entiers, par exemple $1 / 2,2 / 3$ ou $91 / 15$. L'ensemble des nombres réels, noté $\boldsymbol{R}$, contient, en plus de l'ensemble des nombres rationnels, l'ensemble des nombres irrationnels. Ces derniers sont des nombres qui ne peuvent pas s'écrire sous la forme d'un rapport de deux nombres entiers. Par exemple, $\sqrt{2}$, le nombre $\pi$, ou encore le nombre d'Euler $e$ sont des nombres irrationnels.

${ }^{3}$ Par exemple, si le temps est représenté comme discret, il n'y a pas d'instant $t_{3}$ compris entre les instants $t_{1}=1$ et $t_{2}=2$.

${ }^{4}$ Pour un exposé historique sur la construction des nombres réels, voir par exemple (Belna, 1996).
} 
dire que l'on peut associer un nombre entier à chaque nombre rationnel, l'ensemble des nombres réels est indénombrable.

La différence entre continuité et simple densité n'a pas toujours été considérée comme une distinction philosophique majeure. Selon Russell, la continuité, au sens où l'on vient de la définir, est une notion technique des mathématiques alors que la notion de densité, seule, est une notion philosophique importante :

Les mathématiciens ont distingué divers degrés de continuité et ont fait du terme «continu» un terme technique réservé à des séries ayant un degré très élevé de continuité. Mais pour la recherche philosophique, tout ce qu'il y a d'important dans la continuité s'introduit au degré le moins élevé de la continuité, qui s'appelle le caractère «compact». On dit qu'une série est «compacte» lorsqu'il n'existe pas de paire de termes dont l'un soit le successeur immédiat de l'autre, mais qu'au contraire, il existe toujours d'autres termes entre eux. (Russell, 1971, p. 144)

Le terme «compact» est synonyme ici de «dense» : il s'agit de la propriété selon laquelle, entre deux éléments quelconques d'une série ou d'un ensemble - tel que l'ensemble des rationnels - il existe toujours un terme compris entre les deux. Une autre manière de caractériser cette propriété est, comme le fait Russell dans l'extrait précédent, d'énoncer que pour tout élément d'un ensemble ou d'une série dense, il n'existe pas de successeur. La densité se distingue sur ce point du caractère discret. Alors que chaque nombre entier possède un successeur, par exemple 2 est le successeur de 1, il n'existe pas de successeur au nombre 1 dans l'ensemble des nombres rationnel.

Je fais l'hypothèse que Russell privilégie le concept de densité sur celui de continuité car cette première notion suffit à rendre compte de la divisibilité à l'infini du temps et de l'espace. Il s'agit d'une notion qui est notamment mobilisée dans les paradoxes de Zénon que Russell examine quelques pages plus loin (Russell, 1971, p. 176). Par exemple, le paradoxe de la dichotomie selon lequel une longueur peut toujours être divisée en deux peut être énoncé à l'aide de la seule notion de densité. Les divisions successives d'une longueur unité en $1 / 2$ puis $1 / 4$ puis $1 / 8$ etc. ne mobilisent que des nombres rationnels. Le but de cet article n'est pas d'engager une discussion sur les paradoxes de Zénon. En revanche, contre ce que suggère Russell, je défends que la notion de continuité n'est pas seulement une notion mathématique technique mais doit aussi être prise au sérieux en philosophie. En effet, les théories physiques traditionnelles utilisent la représentation du temps comme continu et non pas comme seulement dense. Dès lors, les philosophes qui s'intéressent aux théories physiques utilisées par les scientifiques doivent s'interroger sur la représentation du temps telle qu'elle est utilisée par les scientifiques. C'est précisément dans le but de montrer que la représentation traditionnelle du temps comme continu n'est pas la seule représentation 
envisageable du temps que Newton-Smith s'intéresse à la construction de la mécanique de Notwen.

\subsection{La mécanique dense}

Le but poursuivi par Newton-Smith est de montrer que l'on peut construire une mécanique classique en représentant le temps comme simplement dense. Pour cela, il commence par identifier comment le recours à la représentation continue du temps se manifeste dans la mécanique classique de Newton. Cette représentation se retrouve dans les définitions des notions de vitesse et d'accélération instantanées. La vitesse instantanée est définie comme la dérivée de la position par rapport au temps, $v(t)=d x / d t$, où le paramètre $t$ est défini sur un intervalle de nombres réels. En effet, la vitesse est définie comme la limite quand $h$ tend vers zéro, du rapport $(x(t+h)-x(t)) / h$ où $x(t)$ représente la position du corps à l'instant $t$, et $h$ un intervalle de temps avec $h$ un nombre réel. De même, l'accélération instantanée est définie comme la dérivée de la vitesse par rapport au temps. Ainsi, le principe fondamental de la mécanique de Newton, selon lequel le produit de la masse d'un corps par son accélération est égal à la somme des forces, s'écrit sous la forme de l'équation différentielle $m d v / d t=F$ et utilise une représentation du temps comme continu.

Recourir à une représentation dense du temps suppose de se passer des définitions précédentes. Newton-Smith propose de prendre pour concepts fondamentaux de sa mécanique de Notwen, non plus les notions de vitesses et d'accélérations instantanées mais celles de vitesses et d'accélérations moyennes et de remplacer les équations différentielles par des équations aux différences. En choisissant de représenter le temps comme dense, NewtonSmith définit la notion de vitesse moyenne comme $v_{\text {moy }}(t)=(x(t+h)-x(t)) / h$ où, cette fois, $t, h$ et $x$ appartiennent à l'ensemble des nombres rationnels. De la même manière, il définit une notion d'accélération moyenne comme $a_{\text {moy }}(t)=\left(v_{\text {moy }}(t+h)-v_{\text {moy }}(t)\right) / h$.

Dans ces conditions, le principe fondamental de la dynamique de la mécanique de Notwen se réécrit comme $m a_{m o y}(t)=F$, c'est-à-dire sous la forme de l'équation aux différences suivante : $m(x(t+2 h)-2 x(t+h)+x(t)) / h^{2}=F$. Le cas d'étude sur lequel Newton-Smith fonde sa discussion consiste ainsi à reformuler, à l'aide d'un paramètre temporel parcourant l'ensemble des nombres rationnels, la mécanique classique de Newton en remplaçant les équations différentielles par des équations aux différences définies sur des nombres rationnels. Cette mécanique conserve les mêmes lois et principes que ceux de la mécanique de Newton. Elle diffère en revanche de la mécanique classique traditionnelle par l'élimination du recours aux nombres irrationnels dans la représentation du temps.

La construction de cette mécanique dense repose sur une proposition que l'on retrouve chez Carnap et Putnam, à savoir que les résultats de mesure en physique ne sont jamais des nombres irrationnels et, qu'à ce titre, il est envisageable que la physique puisse s'en passer. 
Dans Les fondements philosophiques de la physique, Carnap pose en effet la question de savoir si l'on peut se passer des nombres irrationnels en physique. Ces nombres, tels que $\pi$ ou $\sqrt{ } 2$, ont pour particularité de ne pouvoir s'écrire à l'aide d'un nombre fini de décimales ${ }^{5}$ et, par conséquent, ne peuvent jamais être des résultats numériques d'une mesure mais seulement les résultats d'un calcul :

on considère généralement que la classe des valeurs possibles d'une grandeur physique contient tous les nombres réels, ce qui inclut les irrationnels en sus des nombres rationnels. Cependant, ces nombres irrationnels sont introduits à un stade ultérieur à celui des opérations de mesure. La mesure directe ne peut fournir que des valeurs exprimées en nombres rationnels. [...]

Comme les nombres irrationnels résultent toujours d'un calcul et non pas d'une mensuration directe, ne serait-il pas possible en physique d'abandonner entièrement les nombres irrationnels et de travailler avec les seuls nombres rationnels? Ce serait en effet possible, mais le changement serait révolutionnaire. Par exemple, il deviendrait impossible d'utiliser les équations différentielles, parce qu'elles requièrent le continuum des nombres réels. (Carnap, 1973, p. 91 et p. 92)

Carnap illustre son propos sur l'exemple de la diagonale d'un carré de côté unité. La longueur $\sqrt{ } 2$ est le résultat d'un calcul déduit, par exemple, du théorème de Pythagore. En revanche, le résultat numérique de la mesure de la diagonale du carré ne sera jamais qu'un nombre avec un nombre fini de décimales. Ainsi, si le recours aux nombres en physique se limite à des résultats de mesures, on peut en effet supposer que les nombres irrationnels ne soient pas indispensables en physique.

Les philosophes des sciences ne se sont pas limités à défendre la possibilité de se passer des nombres irrationnels pour les seules mesures physiques. Ils ont avancé que l'on pouvait aussi s'en passer pour énoncer les lois fondamentales des théories physiques :

au premier abord, la loi de la gravitation - que nous prétendons être la seule loi de la physique au cours de cet essai - nécessite une quantification sur des nombres réels. Cependant, cette dernière loi est équivalente à la proposition suivante : pour chaque rationnel $\varepsilon$ et tous les rationnels $m_{1}, m_{2}$ et $d_{1}$, il existe un rationnel $\delta$ tel que :

Si $M_{a}=m_{1} \pm \delta, M_{b}=m_{2} \pm \delta, d=d_{1} \pm \delta$ alors $F=\left(g m_{1} m_{2} / d_{1}^{2}\right) \pm \varepsilon$

\footnotetext{
${ }^{5}$ Les nombres irrationnels ne peuvent pas s'écrire à l'aide d'un nombre fini de décimales, et ceci dans n'importe quelle base. Ils se distinguent sur ce point des nombres rationnels tels que $1 / 3$. Si ce dernier s'écrit avec un nombre infini de décimales en base 10 , à savoir $0,3333 \ldots$, il s'écrit en revanche 0,1 en base 3 .
} 
et cette proposition utilise uniquement des quantifications sur des nombres rationnels. (Il subsiste toutefois le problème de la constante de gravitation qui peut ne pas être rationnelle ! Je ne m'en soucierai pas ici). Ainsi, un langage qui quantifie uniquement sur des nombres rationnels et qui mesure les distances, les masses, les forces, etc. à l'aide d'approximations rationnelles («la masse de $a$ est $\left.m_{1} \pm \delta »\right)$ est, en principe, assez puissant pour énoncer au moins la loi de la gravitation. (Putnam, 1971, p. 51)

Dans Philosophie de la logique, Putnam soutient qu'il existe deux énoncés équivalents de la loi de la gravitation. Le premier est l'énoncé mathématique traditionnel selon lequel $F=g M_{a} M_{b} / d^{2}$ où $F, M_{a}, M_{b}$ et $d$ sont des nombres réels. Le second est $F_{0}=g m_{1} m_{2} / d_{1}{ }^{2}$ où $F_{0}$, $m_{1}, m_{2}$ et $d_{1}$ sont des nombres rationnels. Ces deux énoncés sont équivalents au sens suivant. Considérons une grandeur rationnelle $\varepsilon$ aussi petite que l'on veut. Dans ces conditions, il est toujours possible d'assigner des valeurs rationnelles aux masses des deux corps et à leur distance qui diffèrent au plus d'une quantité rationnelle $\delta$ par rapport aux masses $M_{a}$ et $M_{b}$ et à la distance $d$, telles que la différence entre les forces $F$ et $F_{0}$ soit plus petite que $\varepsilon$. Dans ces conditions, si $\varepsilon$ et $\delta$ sont plus petits que la précision des mesures possibles pour les forces, masses et distances, aucune mesure ne permet de distinguer la force $F$ formulée avec les nombres réels de la force $F_{0}$ formulée avec les seuls nombres rationnels.

Carnap et Putnam envisagent ainsi la possibilité de se passer des nombres irrationnels en physique. Mais ils ne font que suggérer cette idée. Newton-Smith, au contraire, cherche à déployer cette proposition en exemplifiant une mécanique dense «empiriquement équivalente» (Newton-Smith, 1980, p. 123) à la mécanique de Newton.

\subsection{Equivalence empirique}

Par empiriquement équivalent, Newton-Smith entend qu'il n'est pas possible de distinguer par des mesures physiques une prédiction établie à partir de la mécanique de Newton d'une prédiction établie à partir de la mécanique de Notwen. Cette équivalence empirique est justifiée en interprétant les équations aux différences comme des approximations aussi proches que l'on veut des équations différentielles :

Par exemple, la loi [de Notwen] pour la force est la suivante : $F=m(x(t+2 h)-2 x(t$ $+h)+x(t)) / h^{2}$ où $h$ représente un intervalle rationnel. Cette équation devient une approximation aussi proche que l'on veut de $F=m d^{2} x / d t^{2}$ en prenant $h$ suffisamment petit. En effet, la mécanique de Notwen recourt aux notions de vitesse et d'accélération moyennes et non à celles de vitesse et d'accélération instantanées. Le $h$ dans l'équation précédente représente un intervalle rationnel sur lequel les moyennes sont définies. En prenant $h$ suffisamment petit, les 
équations de Notwen sont des approximations aussi proches que l'on veut des équations newtoniennes. (Newton-Smith, 1980, p. 122)

Selon Newton-Smith, en prenant $h$ aussi petit que l'on veut, les notions de vitesse et accélération moyennes de la mécanique dense sont des approximations aussi proches que l'on veut des notions de vitesse et accélération instantanées de la mécanique de Newton.

Cet argument mérite d'être davantage explicité que ne le fait Newton-Smith. Il faut distinguer trois notions de vitesse : (i) la notion de vitesse instantanée définie dans la mécanique de Newton que je note $v$, (ii) une notion de vitesse moyenne définie dans la mécanique de Newton que je note $v_{c, m o y}$ et (iii) la vitesse moyenne définie dans le cadre de la mécanique de Notwen, notée $v_{\text {moy }}$. La vitesse moyenne de la mécanique de Newton est définie comme $v_{c, m o y}(t)=(x(t+h)-x(t)) / h$ où $t, h$ et $x$ appartiennent à l'ensemble des nombres réels. On passe de cette vitesse à la vitesse instantanée en prenant la limite quand l'intervalle de temps réel $h$ tend vers zéro. En prenant un intervalle de temps $h$ fini et réel aussi petit que l'on veut, la vitesse moyenne est une approximation aussi proche que l'on veut de la notion de vitesse instantanée. Cela se justifie notamment en montrant que la différence entre ces deux vitesses est, lorsque $h$ est suffisamment petit, proportionnelle à $h$. Ensuite, il faut justifier que la vitesse moyenne de la mécanique dense est une approximation aussi proche que l'on veut de la vitesse moyenne de la mécanique de Newton. Cela se justifie par la propriété de densité. L'ensemble des nombres rationnels étant dense dans l'ensemble des nombres réels, pour tout nombre réel $x_{\mathrm{R}}$, il existe un nombre rationnel $x_{\mathrm{Q}}$ aussi proche que l'on veut de $x_{\mathrm{R}}$. Dans ces conditions, il devient possible de définir une vitesse moyenne avec des paramètres rationnels, aussi proche que l'on veut de la vitesse instantanée de la mécanique de Newton.

À partir de cette relation d'approximation, Newton-Smith conclut que les prédictions établies à partir de chacune de ces deux mécaniques ne peuvent pas être distinguées empiriquement. Son argument est le suivant. Il commence par considérer qu'une prédiction en mécanique consiste en la prédiction de la valeur numérique d'une variable, par exemple celle de la distance $x(t)$ à laquelle se trouve un corps à un instant $t$. Il fait ensuite remarquer que le résultat d'une telle prédiction établie dans le cadre de la mécanique dense est un nombre rationnel, que je note $x_{\mathrm{Q}}$. En revanche, le résultat de la prédiction établie dans le cadre de la mécanique de Newton est un nombre réel que je note $x_{\mathrm{R}}$. En prenant $h$ aussi petit que l'on veut dans les équations fondamentales de la mécanique de Notwen, les prédictions $x_{\mathrm{R}}$ et $x_{\mathrm{Q}}$ sont, selon Newton-Smith, aussi proches que l'on veut les unes des autres. À ce moment intervient un second élément dans son argumentation. Le résultat numérique d'une mesure physique se présente toujours comme un nombre rationnel et jamais comme un nombre irrationnel. Il s'agit de l'argument utilisé par Carnap et Putnam pour justifier que l'on peut se passer des nombres irrationnels pour les mesures physiques. Dans ces conditions, les prédictions $x_{\mathrm{Q}}$ et $x_{\mathrm{R}}$ ne peuvent pas être distinguées empiriquement : 
La théorie de Notwen prédit que le paramètre a une valeur rationnelle, celle qui est le résultat d'une mesure empirique; la théorie de Newton peut prédire que le paramètre a une valeur irrationnelle pour laquelle la valeur mesurée est regardée comme une approximation. Par conséquent, il n'y aura (même en principe) aucune mesure en faveur de [la théorie de] Notwen et qui peut être contre [celle de] Newton ou en faveur de celle de Newton et contre [celle de] Notwen. [...] Toutes les mesures pertinentes qui pourraient être faites laisseront le choix entre les théories sous-déterminées. Toute observation qui confirme l'une, confirme l'autre et toute observation qui falsifie l'une, falsifie l'autre. (Newton-Smith, 1980, p. 124)

Newton-Smith justifie ainsi l'équivalence empirique entre les deux théories à la manière de Carnap et Putnam. Il soutient que ce qui différencie les deux mécaniques - à savoir que l'une prédit une valeur réelle et l'autre une valeur rationnelle - n'est pas susceptible d'être mis en évidence par des mesures physiques. Dans la section suivante, je montre que cette équivalence empirique n'est pas suffisamment convaincante avant de proposer cependant une solution pour pallier cette critique.

\section{Mécanique dense et théorie utilisable}

La construction de la mécanique de Notwen proposée par Newton-Smith n'est pas satisfaisante au sens où elle s'avère inutilisable par les scientifiques. Elle conduit à une théorie physique qui ne permet pas d'établir des prédictions effectives sur les phénomènes (section 2.1). En revanche, il est possible de définir une nouvelle représentation dense du temps à partir de laquelle une mécanique dense utilisable peut être construite (section 2.2).

\subsection{La mécanique de Notwen inutilisable}

Newton-Smith justifie l'équivalence empirique de la mécanique de Newton avec celle de Notwen sur la base d'une équivalence empirique de leurs prédictions. Cependant, il ne montre pas comment les scientifiques peuvent établir de telles prédictions. Les équations fondamentales de la mécanique de Notwen sont des équations aux différences finies. NewtonSmith suggère la possibilité du développement d'une mécanique de Notwen empiriquement équivalente en avançant un développement parallèle de la théorie des équations différentielles et de la théorie des équations aux différences :

la théorie des équations aux différences peut être développée d'une manière parallèle à la théorie des équations différentielles. Pour toute équation différentielle définie sur un ensemble continu, il existe une équation aux différences définie sur l'ensemble dense approprié et qui en est une approximation aussi proche que l'on veut. (Newton-Smith, 1980, p. 122) 
Il ne précise cependant pas que la représentation dense du temps qu'il propose conduit à l'utilisation d'un domaine très restreint de la théorie des équations aux différences : les équations aux différences définies sur les nombres rationnels.

Contrairement à l'utilisation restrictive qu'en fait Newton-Smith, la théorie des équations aux différences a généralement recours à l'ensemble des nombres réels, voire à celui des nombres complexes. Se limiter aux seules équations aux différences définies sur les nombres rationnels limite sévèrement - voire rend impossible - l'utilisation de la théorie des équations aux différences. Elle empêche, par exemple, le recours aux fonctions mathématiques définies sur l'ensemble des nombres réels telles que les fonctions racine carré, sinusoïdale, exponentielle etc. Il est par exemple impossible de décrire un phénomène physique avec une mécanique sans les nombres réels si la résolution de l'équation $x^{2}=2$ est requise. Cette équation n'a en effet pas de solution si $x$ est un nombre rationnel.

Considérons l'exemple suivant, celui la chute d'un corps avec une mécanique sans les nombres réels, où $d$, $t$ et $g$ sont des variables et des paramètres rationnels représentant respectivement la distance, la durée de la chute et l'accélération de la pesanteur. En mécanique de Newton, ces grandeurs satisfont la relation $d=g t^{2} / 2$ si le corps a une vitesse initiale nulle. Dans ce cas, la durée de la chute s'écrit en fonction de la distance et de l'accélération de la pesanteur comme $t=\sqrt{ }(2 d / g)$. Cette équation n'a, de manière générale, pas de solution avec une mécanique sans les nombres réels. Par exemple, si $d=g$ alors $t=\sqrt{ } 2$. Cette solution n'est pas définie si le paramètre $t$ est un nombre rationnel.

Dans ces conditions, il est difficile de ne pas interpréter la mécanique de Notwen comme une reformulation artificielle de la mécanique classique. Il est sans doute possible de redéfinir certaines fonctions mathématiques avec les seuls nombres rationnels. Mais, en pratique, l'établissement de prédictions effectives, tel que l'établissement de l'équation de la trajectoire d'un corps en chute libre, semble difficile si l'on se limite aux seuls nombres rationnels. Newton-Smith est conscient de cette difficulté. Il admet lui-même qu'il s'agit ici d'une approche seulement en principe des théories : «si ce n'est en pratique, il existe du moins en principe une théorie rivale à la mécanique newtonienne que j'appellerai la mécanique de Notwen» (Newton-Smith, 1980, p. 123). Cependant, s'il s'avère impossible d'établir des prédictions effectives, on peut douter que la mécanique de Notwen puisse être légitimement considérée comme une théorie physique. La critique que j'adresse ici repose sur une conception des théories physiques qui prend au sérieux la distinction entre les théories scientifiques telles qu'elles peuvent utilisées en pratique par les scientifiques et leurs reconstructions formelles. Selon la conception des théories scientifiques que j'adopte, «une théorie est à la fois un outil de représentation et un outil d'inférence ou de calcul» (Vorms, 2009 , p. 2). Une théorie physique ne se réduit pas à un ensemble d'axiomes ou de modèles. 
Une approche formelle qui ne permettait pas d'établir en pratique des prédictions physiques ne compte pas, selon cette approche, comme une authentique théorie physique.

Newton-Smith tente de devancer cette objection. Selon lui, s'il s'avère trop compliqué ou impossible de résoudre les équations de la mécanique de Notwen sans utiliser les nombres irrationnels, il suggère d'utiliser tous les nombres réels mais de considérer que seuls les nombres rationnels représentent des instants. Il ne s'agit pas d'une réponse plus satisfaisante. On peut en effet se demander ce qu'il reste de la théorie de Notwen si l'on doit utiliser la théorie de Newton pour résoudre les équations de la théorie de Notwen. Il semble en effet qu'une théorie physique, pour compter comme telle, doit posséder une certaine autonomie. Elle doit permettre de décrire et prédire un ensemble de phénomènes, sans pour cela recourir nécessairement à une autre théorie.

\subsection{La représentation dense du temps avec les nombres réels}

Contrairement à ce que semble suggérer Newton-Smith, le recours à une représentation du temps comme dense ne suppose pas forcément l'élimination des nombres irrationnels. Il convient de distinguer deux notions : le recours à une représentation du temps comme dense et la représentation du temps à l'aide d'un paramètre $t$ défini sur l'ensemble des nombres rationnels. Représenter le temps comme dense consiste à représenter le temps à l'aide d'un paramètre défini sur un ensemble dense. Pour la construction de la mécanique Notwen, Newton-Smith utilise l'ensemble des nombres rationnels. Mais il est possible de représenter le temps comme dense tout en utilisant un paramètre réel. Dans ce cas, nous allons voir que la mécanique dense construite à l'aide de cette nouvelle représentation du temps devient utilisable par les scientifiques car les résultats mathématiques requérant les nombres réels peuvent être mobilisés.

Je propose d'adopter la représentation suivante du temps comme dense. Le temps est représenté comme un ensemble -- noté $T$-- d'instants $t_{k}$ défini comme suit. Soit $M$ un nombre réel et $N$ un nombre entier fixés. Définissons un premier ensemble $T_{N}$ de la manière suivante : $T_{N}=\left\{t_{k}=k M / N, k \in N\right.$, tel que $\left.k \leq N\right\}$. Il s'agit d'un ensemble fini de $N+$ léléments $t_{k}$. Par exemple, si $M=2$ et $N=5, T_{5}=\left\{t_{0}=0, t_{1}=2 / 5, t_{2}=4 / 5, t_{3}=6 / 5, t_{4}=8 / 5, t_{5}=2\right\}$. Dans ces conditions, l'ensemble $T$ est défini comme : $T=C_{N \in N} T_{N}$. Il s'agit de l'union des ensembles $T_{N}$ pour tout $N$ appartenant à $N$. $T$ est un ensemble dense de nombre réels mais qui n'est cependant pas un ensemble complet comme l'ensemble des nombre réels. $T$ est en effet un ensemble infini mais dénombrable de nombres réels. Cette caractéristique est plus saillante si l'on réécrit l'ensemble $T$ comme $T=M[0,1] \dot{E} \boldsymbol{Q}$, où $\boldsymbol{Q}$ l'ensemble des nombres rationnels. $T$ est ainsi l'ensemble des nombres réels qui s'écrivent $M$ fois un nombre rationnel compris 
entre 0 et 1 . Il a la même cardinalité que l'ensemble des nombres rationnels $\boldsymbol{Q}$ et, par conséquent, est un ensemble infini et dénombrable ${ }^{6}$.

Il est possible de réécrire l'ensemble $T_{N}$ en introduisant un paramètre réel $h$ défini comme $h=M / N$. Si $M$ représente une durée, $h$ représente le pas de temps séparant $N+1$ instants. Par exemple, si $M=2$ et $N=5$, le pas de temps $h$ est égal à $2 / 5$ et l'ensemble $T_{5}$ est ainsi l'ensemble des six instants $t_{k}$ compris entre 0 et 2 , et espacés du pas de temps $2 / 5$. Dans ces conditions, étant donné un nombre réel $M$, l'ensemble $T$ est un ensemble infini et dénombrable d'instants $t_{k}$ compris entre 0 et $M$. Contrairement au cas des ensembles $T_{N}$, dans l'ensemble $T$, le pas de temps $h$ n'est pas fixé puisque $N$ parcourt l'ensemble des nombres entier $N$. Ainsi, quels que soient deux instants $t_{k}$ et $t^{\prime}{ }_{k}$ appartenant à $T$ avec $t_{k}<t^{\prime}{ }_{k}$, il existe toujours un instant $t{ }^{\prime \prime}{ }_{k}$ compris entre les deux.

En adoptant cette représentation du temps, je propose de construire une nouvelle mécanique dense qui rend possible la formulation de prédictions. Pour cela, je m'appuie sur une reformulation déjà existante de la mécanique classique pour laquelle le temps est représenté de manière discrète (Lee 1983, Marsden et al. 2001). En l'occurrence, le temps est représenté par un paramètre discret $t_{k}=k h$ avec $0 \leq k \leq N$ et $h$ un nombre réel. Cette représentation discrète du temps correspond à la représentation du temps à l'aide de l'ensemble fini d'instants $T_{N}$ défini précédemment et pour lequel $N=M / h$ est fixé ${ }^{7}$. Il s'agit ici d'une représentation discrète du temps qui diffère de la définition de Newton-Smith qui, comme je l'ai mentionné à la section 1.1, se limite à une représentation du temps par un paramètre discret $t$ défini sur l'ensemble des nombres entiers. L'avantage de la représentation discrète utilisée par Lee et Marsden et al. est qu'elle n'élimine pas les nombres réels et, par conséquent, rend possible le recours aux outils de l'analyse mathématique. La mécanique discrète sur laquelle je m'appuie pour construire une mécanique dense a été initialement développée à partir des années 1980 et continue toujours à faire l'objet de recherche ${ }^{8}$. Il s'agit d'une reformulation discrète de la mécanique lagrangienne fondée sur un principe de moindre action discret. Je ne discuterai pas ici les fondements de cette mécanique discrète mais je propose, sur un exemple, de montrer dans quelle mesure la mécanique dense construite sur le

\footnotetext{
${ }^{6}$ On pourrait également remplacer $N$ par $10^{N}$ dans la définition de $T_{N}$. Dans ces conditions, $T$ serait l'ensemble des nombres réels qui s'écrivent $M$ fois un nombre décimal compris entre 0 et 1 . Un nombre décimal est un nombre dont l'écrire décimal fait intervenir un nombre fini de chiffres après la virgule. Comme l'ensemble des nombre rationnel, l'ensemble des nombres décimaux, noté $\boldsymbol{D}$, est un ensemble infini et dénombrable.
}

\footnotetext{
${ }^{7}$ Notons que la valeur de $M$ doit être un multiple de $h$.

${ }^{8}$ Voir par exemple (Lee et al., 2009).
} 
même principe est une mécanique utilisable et empiriquement équivalente à la mécanique continue ${ }^{9}$.

Considérons à nouveau le cas d'un corps en chute libre. La mécanique classique continue permet de décrire la trajectoire du corps et prédire son mouvement. Pour cela, il suffit de résoudre les équations du mouvement. Il est connu que cette trajectoire est une parabole dont l'équation s'écrit : $z(x)=-\left(g / 2 v_{x, 0^{2}}\right) x^{2}+\left(v_{z, 0} / v_{x, 0}\right) x$ où $z$ représente l'altitude du corps, $x$ sa distance, $g$ l'accélération de la pesanteur, $v_{x, 0}$ et $v_{z, 0}$ les composantes de la vitesse initiale selon les axes horizontaux et verticaux. La résolution des équations de la mécanique discrète permet, de la même manière, de déduire la trajectoire d'un corps en chute libre. Dans ce cas, il s'agit de résoudre des équations aux différences et dont la solution s'écrit : $z_{k}\left(x_{k}\right)=-$ $\left(g / 2 v^{2} x, 0\right) x_{k}^{2}+\left(\left(v_{z, 0}+g h\right) / v_{x, 0}\right) x_{k}$ où, $x_{k}$ et $z_{k}$ représentent respectivement la distance et l'altitude du corps à l'instant $t_{k}$ appartenant à l'ensemble $T_{N}$ et $h$ un pas de temps fixé ${ }^{10}$. Dans le cas de la mécanique dense, l'ensemble des instants $T$ ne suppose plus un pas de temps $h$ fixé. Elle se distingue sur ce point, comme nous l'avons vu, de la mécanique discrète. Contrairement à l'ensemble $T_{N}$ de la mécanique discrète, l'ensemble $T$ est un ensemble infini.

Dans le cas de la mécanique discrète, la trajectoire du corps en chute libre est une série de points $\left(x_{k}, z_{k}\right)$ le long d'une parabole. Cette parabole n'est pas celle déduite de la mécanique continue puisqu'elle en diffère d'un terme «gh». Mais, dans le cas de la mécanique dense, étant donné que le pas de temps $h$ n'est pas fixé, ces deux paraboles sont aussi proches que l'on veut l'une de l'autre. Ce comportement est garanti par le fait que, dans la limite où le pas de temps tend vers zéro, l'équation de la trajectoire de la mécanique discrète tend vers celle de la mécanique continue. Dans ces conditions, il n'est pas possible de discriminer empiriquement la prédiction de la trajectoire de la mécanique continue de celle de la mécanique dense à l'aide d'une mesure physique. En effet, quelle que soit la précision des mesures de positions, il existe toujours une valeur pour le pas de temps $h$ en-dessous de laquelle la différence entre ces deux prédictions est plus petite que la précision de la mesure. Ces deux prédictions sont sous-déterminées par les mesures de position. Ce résultat se généralise aux solutions des équations de cette mécanique dense pour n'importe quel système. Cette mécanique dense est ainsi une candidate bien plus satisfaisante que la mécanique de Notwen pour discuter les conséquences du «résultat d'indécidabilité» énoncé par NewtonSmith.

\section{Structure et représentation du temps}

\footnotetext{
9 Pour une présentation de cette mécanique discrète et une discussion sur les conséquences quant à la représentation du temps dans les théories physiques, voir (Ardourel et Barberousse, à venir).

${ }^{10}$ Ces résultats sont établis à partir des travaux de D’Innocenzo et al. (1987, p. 247).
} 
Nous venons de voir comment modifier la proposition de Newton-Smith et construire une mécanique dense empiriquement équivalente à la mécanique continue avec laquelle il est possible d'établir des prédictions. Dans la suite de l'article, je propose d'examiner les conséquences que l'on peut tirer de ce résultat d'indécidabilité, à savoir qu'il est impossible de trancher entre deux théories reposant sur des représentations dense et continue du temps. Selon Newton-Smith, ce résultat conduit à deux interprétations possibles des énoncés qui portent sur la structure du temps :

Comment devrions-nous répondre à ce résultat d'indécidabilité? Deux réponses au moins méritent d'être sérieusement considérées. Premièrement, on pourrait simplement conclure que l'argument montre une limite possible à l'étendue de la connaissance humaine. Soit le monde est tel qu'il est vrai que le temps est continu, soit le monde est tel qu'il est vrai que le temps est simplement dense. [...] Je l'appellerai la réponse d'ignorance. La structure continue ou dense du temps est une question empirique à laquelle nous ne pouvons pas avoir de réponse. Il y a aussi ce que j'appellerai une réponse d'arrogance. [...] Selon cette réponse, les énoncés «le temps est continu» et «le temps est dense» ne sont pas considérés comme ayant une signification permettant de faire des conjectures à propos des faits. Ils ne sont pas considérés à proprement parler comme étant vrais ou faux. (Newton-Smith, 1980, p. 126)

Selon la première option, les énoncés le temps est continu et le temps est dense sont des énoncés empiriques indécidables. Selon la seconde option, ce sont des énoncés nonempiriques qu'il est possible d'utiliser, l'un ou l'autre, pour décrire les phénomènes.

Dans les deux cas, Newton-Smith considère que l'équivalence empirique de la mécanique dense et de la mécanique continue permet de tirer des conséquences sur la structure du temps, soit comme un énoncé empirique indécidable, soit comme un énoncé nonempirique conventionnel. Dans cette section, je soutiens que ces conséquences sont trop fortes. L'équivalence empirique entre la mécanique dense et la mécanique continue ne conduit qu'à une indétermination sur la représentation du temps et non forcément sur sa structure.

Pour cela, je commence par identifier un présupposé implicitement admis par NewtonSmith selon lequel le recours à une représentation continue du temps dans les théories physique correspond à une hypothèse faite sur la structure du temps (section 3.1). J'explicite ensuite comment Newton-Smith justifie ce présupposé et clarifie la distinction entre représentation et structure du temps (section 3.2). Je plaide ensuite contre l'adoption de ce présupposé et défends une neutralité du recours à une représentation continue du temps sur la structure du temps (section 3.3).

\subsection{L'hypothèse de la structure continue du temps}


Une condition sans doute minimale pour que des considérations sur la représentation du temps puissent avoir des conséquences sur les propriétés attribuées à la structure temps est la suivante : la structure continue du temps est une hypothèse implicitement contenue dans la représentation du temps comme continu. Autrement dit, en utilisant le paramètre $t$ en mécanique classique on admet, au moins implicitement, l'hypothèse selon laquelle la structure du temps est continue.

Ce présupposé semble généralement admis. On constate, par exemple, que la continuité du temps est présentée comme une hypothèse dans certains manuels de mécanique classique. Selon les premières pages du manuel de Kibble et al. :

Les hypothèses les plus fondamentales de la physique sont probablement celles qui concernent les concepts d'espace et de temps. Nous supposerons que l'espace et le temps sont continus [...]. Ces hypothèses interviennent dans toutes les branches de la physique et, bien qu'elles aient fait l'objet de questionnement, il n'y a aucune preuve convaincante que nous ayons atteint les limites de leur domaine de validité. (Kibble et al., 2009, p. 2)

Deux pages plus loin et sans en justifier davantage l'emploi, le temps est représenté par un paramètre $t$ défini sur l'intervalle réel. Si l'on s'en tient à ce manuel, on peut supposer que la représentation du temps comme continu traduit une hypothèse sur la structure du temps. Maddy énonce encore plus clairement l'idée selon laquelle la continuité du temps est une hypothèse implicitement admise lorsque le temps est représenté comme continu dans les théories physiques. Au chapitre «Indispensabilité et pratique scientifique» de Naturalisme en mathématiques, elle examine les conséquences du recours à la représentation du temps par le paramètre réel $t$ dans les théories physiques. Au cours de cette analyse, elle affirme que le recours à cette représentation du temps contient implicitement l'hypothèse selon laquelle «le temps est continu» (Maddy, 1997, p. 155).

Selon certaines approches, l'hypothèse de la continuité du temps est non seulement implicitement contenue dans la représentation du temps comme continu mais l'hypothèse et la représentation sont même confondues. C'est ce que révèle la reconstruction axiomatique de la mécanique classique proposée par Suppes. La représentation continue du temps est présentée comme un axiome de la mécanique classique. Il s'agit de l'axiome «P2» selon lequel : «l'ensemble $T$ [pour temps] est un intervalle de nombres réels» (Suppes, 1957, p. 294). Il n’y a pas dans ce cas de distinction entre la représentation du temps par un paramètre $t$ défini sur un intervalle réel et l'hypothèse, plus précisément ici l'axiome, selon lequel le temps est continu.

Ceux qui estiment que l'hypothèse de la continuité du temps est implicitement contenue dans la représentation du temps à l'aide du paramètre $t$ défini sur l'intervalle réel, 
explicitent cependant rarement ce qu'ils entendent par cette hypothèse. Quelle signification doit-on attribuer à l'hypothèse selon laquelle le temps est continu? Kibble et al.. et Maddy ne s'y attardent pas. En revanche, Newton-Smith prend soin d'expliciter cette hypothèse : «si le temps est continu alors l'ensemble de tous les instants avec la relation d'ordre "être avant" sera isomorphe à l'ensemble des nombres réels» (Newton-Smith, 1980, p. 114). La structure du temps est définie avec un langage ensembliste et l'hypothèse de sa continuité est présentée comme une propriété de cet ensemble. L'hypothèse d'un temps continu est définie comme un ensemble d'instants que l'on peut mettre en relation biunivoque avec l'ensemble des nombres réels. Autrement dit, lorsqu'on suppose que le temps est continu, on suppose qu'il est un ensemble d'instants ayant les mêmes propriétés mathématiques que l'ensemble des nombres réels. Bunge propose aussi une clarification de l'énoncé le temps est continu au sein d'un cadre plus général, à savoir l'établissement d'une théorie du temps physique (Bunge, 1968). Selon la théorie qu'il propose, le temps physique est une notion fonctionnelle. Un instant -noté $T\left(e, e^{\prime}, k, s\right)$-- est une relation entre deux évènements $e$ et $e$ ', un référentiel $k$ et une échelle chronométrique $s$. Il est associé à un paramètre réel $t$ tel que $T(e, e, k, s)=t$. Dans ce contexte, la continuité du temps, qui apparait dans le troisième axiome de sa théorie, correspond à l'énoncé selon lequel l'ensemble des évènements est isomorphe à l'ensemble des nombres réels, puisque " pour tout évènement $e$, référentiel $k$, échelle chronométrique $s$ et pour tout nombre réel $t^{11}$, il existe un autre évènement $e^{\prime}$ tel que $T\left(e, e^{\prime}, k, s\right)=t$ (Bunge, 1968, p. 359). Autrement dit, relativement à un évènement $e$, pour tout nombre réel $t$, il existe un évènement $e$ ' auquel il est associé.

L'approche proposée par Newton-Smith, comme celle proposée par Bunge, attribue ainsi de nombreuses déterminations mathématiques à l'énoncé le temps est continu. Toutes les propriétés mathématiques de l'ensemble des nombres réels, telles que sa cardinalité infinie et indénombrable ou encore sa complétude sont attribuées au temps. Cela résulte du présupposé selon lequel le recours à la représentation du temps en physique par un paramètre réel $t$ correspond à une hypothèse sur la structure du temps. Dans la section suivante 3.2, j'examine comment Newton-Smith justifie ce présupposé et clarifie à cette occasion la distinction entre représentation et structure du temps. Ensuite, dans la section 3.3, je défends la position selon laquelle le recours à la représentation continue du temps en physique ne doit pas nécessairement être interprété comme une hypothèse sur la structure du temps.

\subsection{De la représentation du temps à la structure du temps}

Selon Newton-Smith, à la question de savoir pourquoi nous supposons une structure continue au temps :

\footnotetext{
${ }^{11}$ Je souligne.
} 
la réponse est simplement que les meilleures théories physiques que nous avons en pratique construites pour décrire le monde physique requièrent dans leur formulation mathématique un paramètre temporel qui parcourt l'ensemble des nombres réels [...]. Chaque nombre réel est supposé dénoter un instant et une infinité indénombrable d'instants est associé à n'importe quel intervalle de nombre réels. [...] Notre croyance en la continuité du temps [...] provient de notre projection sur le monde de la richesse du système mathématique que nous avons considéré jusqu'à maintenant comme étant essentiel à la construction des théories physiques viables. (Newton-Smith, 1980, p. 118)

L'hypothèse de la structure continue du temps est ainsi justifiée par l'idée selon laquelle les théories physiques contemporaines les mieux confirmées utilisent une représentation continue du temps. Par conséquent, et de manière réciproque, «si nos meilleures théories s'avéraient finalement représenter le temps par une variable discrète [ou dense], nous aurions au moins autant de raison de regarder le temps comme discret [ou dense], que nous en avons de le regarder comme continu» (Newton-Smith, 1980, p. 118).

On peut cependant être septique face à cette justification de la structure continue du temps. Il semble possible de reconnaître pleinement le succès des théories physiques représentant le temps comme continu sans forcément considérer le temps comme «une infinité indénombrable d'instants». En effet, les théories physiques contemporaines recourent à une représentation continue du temps dans le but d'étudier les phénomènes qui s'y déroulent, mais non dans le but d'étudier la structure elle-même du temps. Par conséquent, la représentation du temps comme continue pourrait n'être qu'un cadre général pour décrire les phénomènes et ne correspondre à aucune hypothèse spécifique sur la structure du temps.

Avant d'envisager dans la section 3.3 la possibilité de représenter le temps comme continu sans supposer de structure continue au temps, il convient ici de préciser cette distinction entre représentation et structure. La représentation du temps comme continu est, nous l'avons vu, l'utilisation du paramètre réel $t$ pour décrire l'évolution des phénomènes. Cette représentation possède aussi une structure. Mais il s'agit d'une structure mathématique, à savoir celle d'un ensemble de nombres muni de propriétés mathématiques : relation d'ordre, cardinalité infinie et indénombrable, complétude. En revanche, la structure continue du temps est une notion physique. On retrouve cette distinction chez Bunge dans le cadre de la théorie du temps physique qu'il développe. Il souligne que «le concept de temps n'est pas un concept mathématique bien qu'il puisse être mathématisé» (Bunge, 1968, p. 359). Deux symboles différents sont d'ailleurs utilisés dans sa théorie : $T\left(e, e^{\prime}, k, s\right)$ pour l'instant physique et $t$ pour le nombre réel. Le temps étant un concept mathématisé, un ensemble de nombre lui est associé, comme dans le cas du concept mathématisé de masse. Mais cela ne signifie pas que le concept de temps, comme celui de masse, soit un concept mathématique. Comme nous 
l'avons vu, Newton-Smith distingue aussi la représentation de la structure du temps. Alors que la représentation continue du temps est le paramètre $t$ défini sur l'ensemble des nombres réel, la structure du temps est celle d'un ensemble isomorphe à l'ensemble des nombres réels. Il s'agit bien de deux ensembles différents.

L'hypothèse selon laquelle le temps serait un ensemble isomorphe à l'ensemble des nombres réels peut paraître audacieuse. On peut même avant tout douter du présupposé selon lequel la structure du temps serait celle d'un ensemble ${ }^{12}$. Cependant, la critique que j'adresse ici concerne davantage le passage du recours à la représentation continue du temps à l'hypothèse d'une structure continue. Comme nous venons de le voir, la structure du temps n'est pas un objet d'enquête dans les théories physiques. La représentation continue du temps pourrait n'être qu'un cadre pour décrire les phénomènes physiques sans être la traduction mathématique d'une hypothèse des théories physiques.

\subsection{La représentation continue du temps sans la continuité du temps}

Contrairement aux positions adoptées par Kibble et al., Maddy, Newton-Smith ou Suppes, je défends une neutralité du recours à la représentation continue du temps relativement à la structure du temps dans les théories physiques. Cette neutralité est sans doute plus saillante lorsque le temps est représenté à l'aide d'un paramètre $t$ défini sur d'autres ensembles que celui des nombres réels. Considérons, par exemple, la représentation du temps à l'aide d'un paramètre $t$ défini sur l'intervalle de nombres complexes. Il s'agit d'une représentation utilisée pour traiter certains problèmes de physique statistique à partir du formalisme de l'intégrale de chemin. Dans ce cas, nous ne considérions légitimement pas que cette représentation suppose une structure complexe ou imaginaire du temps. De la même manière, lorsque Lee et al. présentent la mécanique discrète évoquée à la section 2.2 , ils ne font à aucun moment référence à l'hypothèse d'une structure discrète du temps ${ }^{13}$. Je plaide en faveur d'un traitement similaire pour la représentation continue du temps. Comme pour la représentation complexe, discrète ou dense du temps, le recours à la représentation continue du temps dans les théories physiques ne doit pas être considéré comme l'adoption d'une hypothèse sur la structure du temps.

\footnotetext{
12 Dummett critique l'hypothèse d'une structure du temps isomorphe à l'ensemble des nombres réels tel que les mathématiques classiques le définissent (Dummett, 2000). Il envisage une représentation du temps sur un modèle constructiviste et qui, selon lui, conduit à une conception du temps plus satisfaisante. Je laisse cette discussion de côté car, comme je l'explicite dans le corps du texte, ma critique concerne le passage de la représentation à la structure du temps et non la notion elle-même de structure continue du temps.

13 Ils définissent la mécanique discrète variationnelle comme «une formulation de la mécanique avec un temps discret qui repose sur un principe discret analogue au principe de Hamilton selon lequel la trajectoire effective d'un système est celle qui rend l'action stationnaire» (Lee et al., 2009, p. 2003). Et, dans la suite de l'article, il n'est à aucun moment évoqué l'hypothèse d'une structure discrète du temps mais seulement le recours à une représentation du temps à l'aide d'un paramètre discret.
} 
D'ailleurs, si certains manuels comme celui de Kibble et al. considèrent la continuité du temps comme une hypothèse de la mécanique classique, d'autres suggèrent que le recours à la représentation continue du temps ne présuppose pas cette hypothèse. C'est l'interprétation que je propose du manuel de physique Feynman (1977). Dans ce manuel, l'auteur énonce au chapitre 5 les différentes techniques physiques permettant de mesurer des durées de plus en plus courtes. Après avoir indiqué les limites techniques actuelles, il pose la question suivante à ses étudiants :

Qu'en est-il des temps plus court? Le "temps" existe-il encore à une échelle plus petite ? Peut-on même parler de temps plus court si l'on ne peut pas mesurer - ou peut-être même raisonnablement penser - quelque chose qui se passe en un temps plus court? Peut-être que non. Il y a des questions ouvertes que vous serez amenés à poser et auxquelles vous pourrez peut-être répondre dans les vingt ou trente prochaines années. (Feynman, 1977, p. 5-3)

Feynman avance ici l'hypothèse d'un temps dont la structure ne serait sans doute pas qualifiée de continue : «le "temps" existe-il encore à une échelle plus petite?» s'interroge-t-il. Pourtant, il décrit quelques pages plus loin, au chapitre 8 , le mouvement d'un corps à l'aide d'une représentation du temps comme continu. Il prend l'exemple du mouvement d'un corps en chute libre dont il décrit la distance parcourue à l'aide de la formule $« s=16 t^{2} »$ (Feynman, 1977, p. 8-2) où $t$ est défini sur un intervalle de nombres réels. Comment devons-nous interpréter ces deux passages? Selon moi, Feynman émet au chapitre 5 une hypothèse sur la structure du temps et qui, en l'occurrence, ne serait sans doute pas qualifiée de continue. En revanche, il représente au chapitre 8 le temps comme continu sans considérer que cette représentation présuppose une structure continue du temps.

Cette interprétation s'oppose à celle que propose Maddy pour expliquer la différence dans le traitement du temps entre les chapitres 5 et 8 du manuel de Feynman. Comme nous l'avons vu à la section 3.1, selon elle, le recours à la représentation continue du temps dans les théories physiques présuppose implicitement l'hypothèse d'un temps continu. Cette thèse peut paraître surprenante. Elle semble incompatible avec le passage de Feynman cité précédemment dans lequel, d'un côté, Feynman émet l'hypothèse d'une structure du temps qui ne serait pas continue et, de l'autre, représente pourtant le temps comme continu. Pire, Maddy souligne que la différence des propos de Feynman entre les chapitres 5 et 8 doit être prise au sérieux et non pas mise sur le compte d'un «raccourci un peu rapide d'un texte introductif» (Maddy, 1997, p. 155). En effet, selon elle, Feynman présuppose bien une structure continue au temps dans le chapitre 8 lorsqu'il le représente comme continu. Cependant, si cette hypothèse est implicite, il ne s'agit pas d'une hypothèse que Feynman se sent engagé à adopter : 
Les physiciens utilisent sans problème toutes les mathématiques qui leurs sont pratiques et efficaces [...] sans se soucier des hypothèses sur les structures physiques que ces mathématiques présupposent (par exemple que le temps est continu dans le cas de Feynman). [...] En dépit du rôle central du continu mathématique dans la théorie physique du mouvement, Feynman considère la question de la micro-structure du temps comme largement ouverte. (Maddy, 1997, p. 155)

L'interprétation de Maddy diffère de la mienne. Selon elle, Feynman émet deux hypothèses contraires sur la structure du temps dans son manuel. Au chapitre 8, la structure du temps est supposée continue alors qu'au chapitre 5, il fait l'hypothèse d'une structure du temps qui ne serait pas continue.

Maddy règle cette tension en soutenant que, même si Feynman présuppose implicitement la continuité du temps lorsqu'il le représente comme continu, ce n'est pas une hypothèse à laquelle il adhère avec force :

les hypothèses d'existence mathématique en sciences et les hypothèses correspondantes à propos de la réalité physique [à savoir la structure continue du temps], ne sont pas considérées comme des hypothèses physiques ordinaires comparables d'un point de vue épistémique : les standards de leur introduction sont plus faibles et leur rôle dans le succès des théories manque de force dans la confirmation [...]. (Maddy, 1997, p. 155)

L'hypothèse d'une structure continue du temps est bien supposée lorsque le temps est représenté comme continu dans les théories physiques. Mais, il s'agit d'une hypothèse physique qui n'a pas suffisamment fait l'objet d'enquête empirique de la part de la communauté scientifique et, à ce titre, manque de force épistémique.

Selon moi, il n'y a pas de réelle tension entre les deux chapitres du manuel de Feynman. Au chapitre 5, Feynman admet l'hypothèse d'une structure du temps qui ne serait pas continue - sans pour autant prendre partie en faveur de cette hypothèse et dont il laisse l'issue ouverte. En revanche, au chapitre 8, lorsqu'il représente le temps comme continu, il n'émet aucune hypothèse sur la structure du temps.

\section{La représentation continue du temps comme cadre théorique}

Je propose maintenant d'expliciter les conséquences que l'on peut légitimement tirer de l'équivalence empirique entre la mécanique continue et la mécanique dense. Au regard de la neutralité défendue à section précédente entre la représentation du temps et la structure du temps, je défends qu'il s'agit de conséquences sur la représentation du temps qui, seules, peuvent être tirées du résultat d'indécidabilité. La représentation du temps comme continu 
doit être interprétée comme un cadre théorique que les scientifiques ont le choix d'adopter pour décrire les phénomènes physiques.

Je commence par expliciter la position de Newton-Smith selon laquelle le résultat d'indécidabilité conduit à interpréter la structure du temps comme un énoncé non-empirique, un cadre théorique pour décrire les phénomènes (section 4.1). Ensuite, à la lumière de l'analyse de la section 3, je défends que les conséquences proposées par Newton-Smith doivent être affaiblies et limitées à la seule représentation du temps (section 4.2).

\subsection{La structure du temps comme cadre théorique chez Newton-Smith}

Nous avons vu que Newton-Smith propose deux conceptions du temps compatibles avec son résultat d'indécidabilité. D’un côté, la «réponse d'ignorance» (Newton-Smith, 1980, p. 126) consiste à adopter une conception réaliste à propos des hypothèses sur la structure du temps. Le temps est continu ou bien dense mais l'équivalence empirique entre la mécanique continue et la mécanique dense nous empêche de décider entre ces deux énoncés. Newton-Smith prend ses distances avec cette première réponse. Il estime notamment qu'elle confère un caractère trop mystérieux au temps car «elle implique d'accepter la possibilité de faits inaccessibles» (Newton-Smith, 1980, p. 233) ${ }^{14}$.

D'un autre côté, la «réponse d'arrogance» au résultat d'indécidabilité consiste à interpréter les hypothèses d'une structure continue ou dense du temps comme des énoncés non-empiriques :

Opter pour la réponse d'arrogance signifie cesser de considérer les propositions empiriquement indécidables comme exprimant des hypothèses à propos des faits. [...] Une possibilité serait qu'elles soient considérées comme un mode de description ou une structure générale permettant d'exprimer des hypothèses à propos des faits. Par exemple, celui qui asserte que le temps est continu n'est pas en train de faire un énoncé à propos d'un fait mais à propos d'un filet [net] pour saisir les faits. Tous les faits peuvent être énoncés au sein de cette structure mais ils peuvent également être énoncés avec la structure rivale fondée sur le traitement du temps comme simplement dense. (Newton-Smith, 1980, p. 235)

Newton-Smith fait appel explicitement à la conception de cadre théorique proposée par Carnap : «celui qui opte pour une conception du temps comme une cadre [framework] théorique est engagé dans quelque chose comme la conception de l'ontologie selon Carnap,

\footnotetext{
14 Newton-Smith ne soutient cependant pas une position anti-réaliste à propos du temps. Il est enclin à adopter une position réaliste dans les contextes où il n'y a justement pas d'indécidabilité. En revanche, lorsqu'il y a indétermination - comme dans le cas qui nous intéresse de la structure continue ou dense du temps - il suggère d'adopter la seconde réponse pour cet aspect du temps : voir (Newton-Smith, 1980, p. 241).
} 
en ce qui concerne au moins les assertions à propos du temps» (Newton-Smith, 1980, p. 240). Newton-Smith défend ainsi la thèse selon laquelle le résultat d'indécidabilité nous conduit à adopter une conception de la continuité du temps comme un cadre théorique. Elle nous permet de décrire les phénomènes mécaniques mais ne doit pas être considérée comme une propriété réelle du temps inaccessible empiriquement.

On retrouve en effet cette conception de la structure du temps dans Empirisme, sémantique et ontologie lorsque Carnap soutient que les questions sur la structure de l'espacetemps doivent être interprétées comme des questions sur le cadre adopté pour décrire les phénomènes physiques :

Une question comme : "Y a-t-il (réellement) des points d'espace-temps?" est ambiguë. On peut l'entendre comme une question interne, et alors la réponse positive est naturellement analytique et triviale. Ou on peut l'entendre au sens externe : "Devons-nous introduire telles et telles formes dans notre langage?"; et, dans ce cas, ce n'est pas une question théorique, mais une question pratique, une affaire de décision plutôt que d'assertion, si bien que la formulation proposée serait trompeuse. (Carnap, 1997, p. 323)

La question interne revient à se demander si la notion de points d'espace-temps est utilisée dans les théories physiques. La réponse est affirmative : c'est un constat sur la manière dont sont formulées les théories physiques. La question externe revient à se demander si le recours à la notion de points d'espace-temps est utile pour décrire les phénomènes physiques. La réponse que l'on peut apporter relève dans ce cas d'un choix, selon l'intérêt que les scientifiques ont d'adopter ou non cette notion dans la formulation des théories physiques.

En tant que question externe, la réponse à cette question est, selon Carnap, une affaire de «décision». Elle ne s'impose pas aux scientifiques mais résulte d'un choix. Carnap souligne en effet que le recours aux points d'espace-temps ne s'impose pas obligatoirement dans les théories physiques. Par points d'espace-temps, il entend «un quadruplet de quatre nombres réels» (Carnap, 1997, p. 323) et, dans ces conditions, affirme que «bien qu'il ne soit pas lui-même de nature théorique, notre choix de tels ou tels traits [des points d'espacetemps] nous est suggéré par nos connaissances théoriques, soit logiques, soit factuelles. [...] La restriction aux coordonnées rationnelles n'entrait en conflit avec aucune connaissance expérimentale, parce que le résultat de toute opération de mesure est un nombre rationnel» (Carnap, 1997, p. 323). Ainsi, représenter l'espace-temps comme un ensemble de quadruplets de nombres réels est un choix parmi d'autres pour décrire les phénomènes physiques. Le recours à un ensemble de quadruplets de nombres rationnels est un autre choix possible. De la même manière, selon Newton-Smith, c'est parce que nous avons le choix de représenter le temps comme continu ou comme dense - en utilisant la mécanique de Newton 
ou la mécanique de Notwen - que la structure du temps peut être interprétée comme un cadre théorique.

\subsection{Le choix de la représentation dense du temps}

La reprise par Newton-Smith de la thèse de Carnap me conduit à énoncer une première objection. Il semble difficile de se passer, comme Carnap et Newton-Smith le défendent, des nombres réels dans les théories physiques. Comme nous l'avons vu dans la section 2.1, se priver des nombres irrationnels conduit à des théories physiques inutilisables. La mécanique de Notwen proposée par Newton-Smith n'est pas une théorie physique qui permet de décrire, prédire et expliquer les phénomènes physiques. Carnap reconnaît que se priver des nombres irrationnels poserait de sérieuses difficultés pour formuler les théories physiques. Mais il maintient cependant qu'il s'agit d'un choix en considérant la question du recours aux nombres réels ou aux nombres rationnels comme une affaire de simplicité : «le choix des nombres réels plutôt que des nombres rationnels ou des entiers comme coordonnées n'est pas tant influencé par les faits d'expérience que par des considérations de simplicité mathématique» (Carnap, 1997, p. 323). Selon cette conception, les scientifiques ont le choix du recourir aux nombres réels ou aux nombres rationnels pour représenter les points d'espace-temps. En revanche, les nombres réels forment un cadre théorique qui conduit à des calculs plus simples.

Selon moi, recourir aux nombres réels ou aux seuls nombres rationnels n'est pas une question de simplicité mathématique. Il s'agit avant tout d'une question de faisabilité. Une mécanique classique sans les nombres irrationnels n'est pas une mécanique moins simple mais une mécanique inutilisable. À ce titre, ce n'est pas une mécanique à laquelle les scientifiques ont réellement le choix de recourir. En revanche, comme nous l'avons vu dans la section 2.2, j'ai montré que l'on peut remplacer la représentation continue du temps par une représentation dense utilisant les nombres réels. Il s'agit d'une représentation qui conduit à une mécanique classique utilisable au sens où elle permet d'établir des prédictions. Dans ce nouveau contexte, on peut légitimement considérer que les scientifiques ont le choix de recourir à l'une ou l'autre de ces deux représentations du temps.

Dans ces conditions, quelles conséquences pouvons-nous tirer de ce choix dans la représentation du temps? Dans la section 3, j'ai critiqué les conclusions tirées par NewtonSmith quant à la structure du temps. Mais est-ce à dire que l'équivalence empirique de la mécanique continue et de la mécanique dense est sans conséquence? Selon moi, il nous faut réviser les conséquences défendues par Newton-Smith et interpréter la représentation du temps comme continu elle-même, et non la structure continue du temps, comme un cadre théorique.

Comme nous l'avons vu, Newton-Smith distingue explicitement la représentation du temps comme continu de la structure du temps comme continu. La représentation du temps 
comme continu est le recours à un paramètre $t$ défini sur un intervalle réel. La structure du temps comme continu est l'hypothèse d'un temps défini comme un ensemble d'instants isomorphe à l'ensemble des nombres réels. Or, ce dont les scientifiques peuvent se passer, c'est de la représentation du temps comme continu. C'est la raison pour laquelle elle peut être interprétée comme un cadre théorique: les scientifiques ont le choix de l'utiliser ou non. Mais en conclure, comme le fait Newton-Smith, que la structure du temps est elle-même un cadre théorique, c'est passer de conséquences sur la représentation du temps à des conséquences sur la structure du temps. J'ai plaidé à la section 3 en faveur d'une neutralité de la représentation du temps sur sa structure supposée. Au regard de cette analyse, le saut que fait Newton-Smith n'est pas justifié. Il requiert, contre la thèse que j'ai défendue, d'admettre que la représentation du temps contient implicitement l'hypothèse d'une structure continue. Je propose ainsi de limiter les conséquences de l'équivalence empirique entre la mécanique continue et la mécanique dense à la représentation du temps. Ce sont les représentations dense et continue du temps qui sont des cadres théoriques que les scientifiques ont le choix d'adopter.

Selon cette interprétation, on peut à nouveau se poser la question de la simplicité des cadres théoriques. Mais, dans ce cas, c'est bien la simplicité de la représentation du temps, et non celle de sa structure, dont il est question. En l'occurrence, alors que les scientifiques ont le choix de représenter le temps comme dense ou comme continu, la représentation continue est sans doute plus simple. Et l'on peut légitimement supposer que c'est la raison pour laquelle cette représentation est en pratique adoptée par les scientifiques. Même si la mécanique dense que j'ai proposée est utilisable, elle reste techniquement plus compliquée dans l'établissement des prédictions, notamment parce qu'elle requière la manipulation d'un symbole supplémentaire, le pas de temps $h$, en plus du paramètre temporel $t_{k}$.

\section{Conclusion}

À la question de savoir si la structure du temps est indécidable, je réponds par la négative, non parce qu'elle serait décidable mais parce que l'on ne peut pas légitimement soutenir qu'elle est indécidable. En revanche, ce sont les représentations elles-mêmes du temps, comme continu ou simplement dense, qui sont indécidables.

L'argument avancé par Newton-Smith en faveur de l'indécidabilité de la structure du temps repose sur l'équivalence empirique entre des théories physiques représentant le temps comme continu et comme simplement dense. Dans cet article, après avoir montré comment modifier la construction de Newton-Smith afin d'avoir un cas satisfaisant d'équivalence empirique, j'ai soutenu que l'on ne peut cependant pas en tirer de conséquences sur la structure du temps. Les deux théories empiriquement équivalentes ne diffèrent que du point de vue de leur représentation du temps dont j'ai plaidé en faveur de la neutralité vis-à-vis de la structure du temps. On ne peut inférer des conséquences sur la structure du temps à partir 
d'une équivalence empirique produite seulement par une différence de représentations. Ainsi, alors que Newton-Smith propose d'interpréter son résultat d'indécidabilité en faveur d'une conception de la structure du temps comme un cadre théorique, je soutiens la conception selon laquelle la représentation elle-même du temps comme continu est un cadre théorique que les scientifiques ont le choix d'adopter pour décrire les phénomènes.

\section{Remerciements}

Cet article est une partie de mon travail de thèse dirigée par Anouk Barberousse et Jacques Dubucs. Je les remercie pour leur soutien, leurs remarques et leurs critiques toujours constructives. J'adresse également mes remerciements à l'Université Paris 1 PanthéonSorbonne et l'Université Paris-Sorbonne qui m'ont offert un cadre de travail idéal pendant ma thèse. Je remercie enfin très chaleureusement mon ami Clément Ruef pour nos discussions et ses suggestions pertinentes pour cet article.

\section{Références bibliographiques}

Ardourel, Vincent et Barberousse, Anouk

201X, «The Representation of Time in Discrete Mechanics»

Belna, Jean-Pierre

1996, La notion de nombre chez Dedekind, Cantor, Frege, Paris, Vrin.

Bergström, Lars

1984, «Underdetermination and realism», Erkenntnis, vol. 21, p. 349-365.

Bunge, Mario

1968, «Physical Time: The Objective and Relational Theory», Philosophy of Science, vol. 35, $\mathrm{n}^{\mathrm{o}} 4$, p. 355-388.

Carnap, Rudolph

1997, «Empirisme, sémantique et ontologie», dans Signification et nécessité [1956], trad. F. Rivenc et P. de Rouilhan, Paris, Gallimard, p. 311-335.

Carnap, Rudolph 
1973, Les fondements philosophiques de la physique [1966], trad. Jean-Mathieu Luccioni et Antonia Soulez, Paris, Armand Colin.

D’Innocenzo, A., Renna, L. et Rotelli, P.

1987, «Some Studies in Discrete Mechanics», European Journal of Physics, vol. 8, p. 245-252.

Dummett, Michael

2000, «Is Time a Continuum of Instants?», Philosophy, vol. 75, n² 294, p. 497-515.

Feynman, Richard P., Leighton, Robert B. et Sands, Matthew.

1977, The Feynman Lectures on Physics: Mainly Mechanics, Radiation, and Heat [1963], Reading Massachusetts, Addison-Wesley.

Hesse, Mary

1980, «The Hunt for Scientific Reason», Proceedings of the Biennial Meeting of the Philosophy of Science Association, vol. 2, p. 3-22.

Kibble, Tom W. B. et Berkshire, Frank H.

2009, Classical Mechanics, Londres, Imperial College Press.

Lee, Tsung-Dao

1983, «Can Time Be a Discrete Dynamical Variable?», Physics Letters, vol. 122B, nºs 3-4, p. $217-220$

Lee, Taeyoung, Leok, Melvin et McClamroch, Harris

2009, «Discrete Control Systems», dans Robert A. Meyer, dir. Encyclopedia of Complexity and Systems Science, Springer, p. 2002-2019.

Maddy, Penelope

1997, Naturalism in Mathematics, Oxford, Clarendon Press.

Marsden, Jerrold E. et West Matthew.

2001, «Discrete Mechanics and Variational Integrators», Acta Numerica, vol. 10, p. 357-514.

Newton-Smith, William Herbert

1978, «The Underdetermination of Theory by Data», Proceedings of the Aristotelian Society, vol. 52, p. 71-91. 
Newton-Smith, William Herbert

1980, The Structure of Time, Londres, Routledge \& Kegan Paul.

Newton-Smith, William Herbert

2000, «The Underdetermination of Theory by Data», dans William Herbert Newton-Smith, dir., A Companion to the Philosophy of Science, Malden Massachusetts, Blackwell.

Norton, John D.

2008, «Must Evidence Underdetermine Theory?», dans M. Carrier et al., dir., The Challenge of the Social and Pressure Practice : Science and Values Revisited, Pittsburgh, Pittsburgh University Press, p. 17-44.

Putnam, Hilary

1996, Philosophie de la logique [1971], trad. P. Peccatte, l'éclat.

Quine, Willard Van Orman

1979, «Comments on Newton-Smith», Analysis, vol. 39, n 2, p. 66-67.

Russell, Bertrand

1971, La méthode scientifique en philosophie. Notre connaissance du monde extérieur [1926], trad. P. Devaux, Paris : Payot.

Salmon, Wesley C.

2001, Zeno’s Paradoxes [1970], Indianapolis : Hackett Publishing.

Suppes, Patrick

1999, Introduction to Logic [1957], Mineola New-York, Dover.

Vorms, Marion

2009, Théories, Modes d'Emploi, thèse de doctorat, Université Paris 1 Panthéon-Sorbonne. 